Products and Services In Practice is provided to readers using text and images from the manufacturer, supplier or distributor and does not imply endorsement by $B D J$ In Practice. Normal and prudent research should be exercised before purchase or use of any product mentioned.

Please send product and services news through to David Westgarth, $B D J$ In Practice via: David.Westgarth@bda.org

\section{Greater comfort and choice}

Belmont chairs are available in a choice of upholstery and for those practices wanting to exude luxury there's the Ultrasoft Pro, which now has an additional eight colours added to the range.

As the name suggests, the look and feel of this fabric is lavish and designed with patient comfort in mind. Within this range are 33 colours, enabling you to tailor your colour choices to the rest of your decor.

Whether you want a classic, cool earthy tone, or a warm and vibrant option (if you're big on colour) there's a colour that will suit. New this year (for those in the latter group) is Melon and Rosy Coral. More traditionalists might prefer the calming tones of some of their other new colours such as Agave or Oxford Blue.

Aesthetics obviously need to be matched with functionality, and likewise in this respect, Belmont will not disappoint. You can visit the Belmont showroom to view their chairs virtually. Tours can be taken from any device and you can be accompanied on your tour by one of the sales team if you'd prefer a 'guided tour'.

The showroom is still open for visitors, so if you want to feel the difference in upholstery then you can come in person. You will need to book an appointment for a face-to-face visit, so that all necessary protocols are in place. However, if you're unable to take a seat, come and take a peek, with a digital stroll through their showroom.

For more information contact Stefana atdental@takara.co.uk.

\title{
Help them overcome fear!
}

Did you know that the fear of pain is one of the most common reasons that people are afraid of receiving dental treatment?

By referring to EndoCare, you can help patients with anxieties and dental phobia to receive the treatment they need.

Our team of specialists use only cutting-edge methods to ensure that treatment is as pain-free as possible. Plus, we give patients the option to watch TV or listen to music before and during treatment, helping to put their minds at ease.

Once we've completed treatment we will return your patients to your care with full details of what weve done and the relevant aftercare tips.

For further information call EndoCare on 02072240999 or visit www.endocare. co.uk.

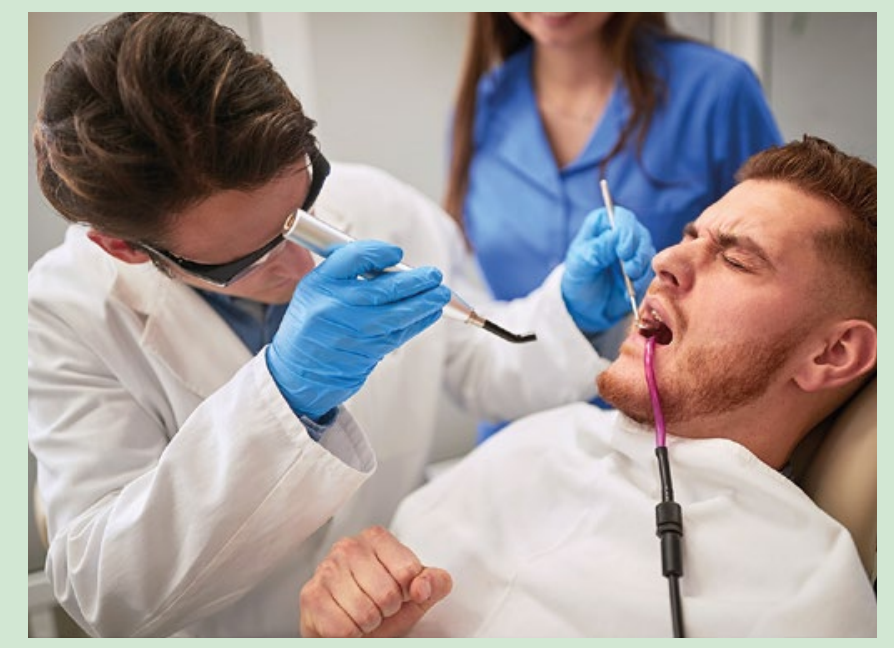

\section{Test for COVID-19 with confidence}

The BioSURE COVID-19 Triple Antibody Rapid Test is an essential diagnostic tool that can support dental teams in continuing to work safely and effectively under the challenges of a global pandemic.

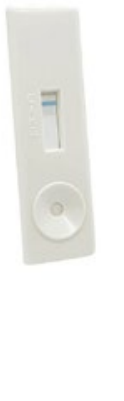

It is a single use, in vitro diagnostic test for COVID-19 antibodies in human blood, with results delivered in just 10 minutes based on a finger prick of blood.

Combined with the BioSURE powered by PocDoc app, this test enables you to assess workforce health and COVID-19 safety across multiple dental practices and over extended periods of time. This supports more efficient planning and better-informed decision-making with regards to getting team members back to work safely.

To order call 01480 862084, emailinfo@curaprox.co.uk or visit www.curaprox.co.uk. 